\title{
L'ÉTHIQUE DE LA RENCONTRE ET LE TRAVAIL DU FANTASME DANS LA FORMATION DES ENSEIGNANTS EN CLASSE D'INCLUSION
}

Jean-Marie Weber

EDP Sciences | «Psychologie Clinique »

2020/2 $n^{\circ} 50 \mid$ pages 84 à 95

ISSN $1145-1882$

Article disponible en ligne à l'adresse :

https://www.cairn.info/revue-psychologie-clinique-2020-2-page-84.htm

Distribution électronique Cairn.info pour EDP Sciences.

(C) EDP Sciences. Tous droits réservés pour tous pays.

La reproduction ou représentation de cet article, notamment par photocopie, n'est autorisée que dans les limites des conditions générales d'utilisation du site ou, le cas échéant, des conditions générales de la licence souscrite par votre établissement. Toute autre reproduction ou représentation, en tout ou partie, sous quelque forme et de quelque manière que ce soit, est interdite sauf accord préalable et écrit de l'éditeur, en dehors des cas prévus par la législation en vigueur en France. Il est précisé que son stockage dans une base de données est également interdit. 


\section{We L'éthique de la rencontre et le travail du fantasme dans la formation des enseignants en classe d'inclusion}

Jean-Marie Weber ${ }^{[1]}$

\section{Résumé}

L'école inclusive constitue un énorme défi pour les enseignants au niveau psychique. Ce texte s'apprête à analyser le défi qui s'ouvre par la rencontre avec l'altérité. Par la présentations de différents extraits d'entretiens, il s'agira d'illustrer les difficultés des futurs enseignants encore en stage à se laisser altérer dans la rencontre avec des élèves à besoins spécifiques. C'est un travail de subjectivation, de déconstruction de leur fantasme qui est nécessaire pour accompagner les jeunes en souffrance. Être à l'écoute de l'autre présuppose d'être à l'écoute de soi-même à travers l'autre. Vouloir rencontrer l'autre dans son altérité est un choix éthique.

\section{Mots clés}

Éthique ; inclusion ; réel ; sujet.

\section{Summary}

Inclusive school is a huge challenge for teachers at the psychic level. This text analyzes the challenge that constitutes the encounter with otherness. Through the presentation of various excerpts from interviews, we will illustrate the difficulties of future teachers still in training to accept themselves to be altered in the encounter with pupils with specific needs. It is a work of subjectivation, of deconstruction of their fantasy that is necessary to accompany young people in suffering. Listening to others presupposes listening to oneself through others. Wanting to meet the other in his otherness is an ethical choice.

Key words

Ethics ; inclusion ; real ; subject.

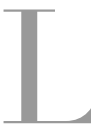

e discours du couple égalité et liberté a conduit à de sensibles efforts d'inclusion de jeunes en difficultés nécessitant une attention spécifique à l'école. Dans ce texte nous voulons aborder les défis et chances que constitue la confrontation avec l'altérité et hétérogénéité pour les jeunes enseignants en formation. Nous nous basons sur des entretiens narratifs conduits avec des enseignants encore en formation à Luxembourg, où les enseignants poursuivent différents stages sous la

[1] Senior lecturer, université du Luxembourg. 
supervision d'un formateur de terrain ou d'un conseiller pédagogique, et en Autriche, où les enseignants poursuivent leur stage sous la supervision d'un “ Praxislehrer» L'analyse des matériaux permet de faire émerger comment le travail de subjectivation qui s’impose dans la rencontre avec ces élèves touche à la dimension du fantasme.

Nous citerons plusieurs propos des enseignants rencontrés, avant de présenter quelques propositions qui nous paraissent incontournables pour la formation des enseignants.

\section{We Le discours de l'inclusion une chance pour l'enfant sujet en difficultés}

Depuis plusieurs décennies l'intégration de jeunes en situation de handicap a été promue dans le domaine associatif, puis à l'école et de plus en plus dans la vie quotidienne. Or le discours de l'intégration, qui constituait un vrai progrès s'est confronté rapidement à ses limites puisqu'il attribuait les difficultés d'une personne à sa déficience, sans distinguer entre les caractéristiques individuelles et les exigences de la société ainsi que les contextes d'action (Garel, 2010). C'est l'avancée introduite par le discours de l'inclusion où une personne est dite " handicapée » en raison d'un côté « de sa déficience qu'elle soit physique, sensorielle, ou mentale, et d'autre part des barrières instrumentales, culturelles sociales, réglementaires créant un obstacle que la personne ne peut franchir en raison de ses particularités» (V. Assante, 2000, p. 8). Ce même discours soutient que la scolarisation réussie d'un enfant « implique de s'attacher dans un même mouvement à ses singularités et à ce qu'il partage, ou peut partager, avec les autres, en particulier une culture commune. Cette attention conjointe aux différences et aux ressemblances invite à conjuguer assimilation et différenciation "(Garel, 2010, p. 160).

Par rapport au discours de l'intégration, le discours de l'inclusion ne répond donc pas seulement aux besoins des enfants, mais il vise à promouvoir à " bien accueillir la diversité »(Konferenz, 2015). Si dans le discours de l'intégration l'enfant avec son mal devait s'adapter à la norme, le discours de l'inclusion vise à adapter l'école aux possibilités de l'enfant. Il s'agit de donner à chacun les mêmes possibilités, de ne pas vivre dans l'exclusion, de développer autant que possible ses potentialités pour que la société, la collectivité puisse elle aussi profiter de cette diversité (Ebersold, 2009). Le discours de l'inclusion exprime donc la volonté politique de promouvoir autant que possible les chances des jeunes dans leur singularité.

La plupart des enseignants acceptent le discours de l'inclusion du point de vue éthique et politique. Mais existent des doutes et des questions sur la mise en pratique dans le quotidien. Ils ont souvent l'impression que la décision politique concernant l'inclusion peut induire des fantasmes d'égalitarisme et des manies de nivellement. Une telle position fait d'ailleurs partie des paradoxes de notre société individualiste qui veut justement aussi exorciser le négatif, l'altérité et admire la "mêmeté » comme l'affirme le philosophe Byung - Chul Han (2016). 
Travaillant depuis plusieurs années dans le domaine de la formation des enseignants à l'université de Luxembourg, j’ai décidé de mener ces entretiens pour essayer de comprendre de plus près comment le jeune enseignant ou l'enseignant-stagiaire de l'école inclusive s'y prend et se forme pour essayer de rencontrer des élèves à besoins spécifiques dans leur singularité et par conséquent dans leur altérité.

\section{$\$$ L'inclusion un défi pour les enseignants (novices)}

\section{档 L'école comme autre scène}

L'école constitue un lieu hautement complexe pour le jeune enseignant. Même s’il y a parcouru toute une carrière d'élève et d'étudiant, il n'est pas nécessairement conscient de tout ce qui se joue à travers la transmission de savoir. En effet, les élèves comme les enseignants y viennent mettre en scène leurs fantasmes, essaient de les éprouver et de leur donner consistance. Et de même pour les parents avec leurs craintes, leurs envies et désirs par rapport à leur progéniture.

Les enseignants eux aussi y vivent leurs désirs, leurs angoisses et haine par rapport aux élèves. Souvent les enseignants ne veulent pas savoir qu'il y a du savoir insu qui circule dans la salle de classe. Or l'enfant, l'adolescent, n'est jamais ce qu'il représente pour les adultes. Ce qui est en jeu pour lui, c'est son advenir en tant que sujet. Son rapport au savoir en porte les traits. C'est ainsi qu'il met en scène son désir ou refus de savoir, ce que ses parents pensent, ont fait ou pas fait. Il se retire dans le mutisme ou encore se marginalise dans un groupe, il fugue pour se protéger de la violence du père, de l'ignorance ou du réel pulsionnel de la mère. Il peut transfèrer sur l'enseignante des attentes qui l'aliènent et dont il souffre.

\section{Confronté au non-savoir que constitue le sujet}

Notre société caractérisée par l'individualisme fait que tout un chacun vit ses propres mythèmes et se retrouve peu structuré par des discours collectifs. Les adultes comme les jeunes se voient livrés à eux-mêmes et souvent à un espace sans bornes. De nouveaux symptômes voient le jour comme l'incapacité de tenir sa place, l'incapacité de faire le choix de son identité sexuelle, une perversion généralisée sous forme d'exhibition impudique, l'usage du pharmakon ou la violence (Melman, 2000). Les enseignants se voient donc confrontés à de nouvelles façons de penser, de jouir et à toute une panoplie de symptômes et d'acting out, et par conséquent à des groupes composés d’individus très hétérogènes.

Travaillant sur les chaines signifiantes et les aspects de jouissance dans les entretiens ou propos lors des supervisions que j’ai pu mener ou lire, nous avons été frappés par le mal que les jeunes enseignants ressentaient en étant confrontés à l'excès, au pulsionnel de l'enfant qu'ils rencontraient. Des enfants ou des jeunes leurs adressent des silences, des peurs, des frustrations, des révoltes, des actes violents. Les enseignants se trouvent en face d'un trop peu de ou un trop de satisfaction, de jouissance. 
Depuis Freud nous savons que si la pulsion c'est la vie, mais c'est aussi la mort quand son trajet tourne en rond. Y faire avec pour un jeune stagiaire ne va pas de soi. Quelqu'un se disait fortement déstabilisé par un jeune qui d'un moment à l'autre " est ailleurs... plus joignable ".

Par association, cet exemple nous renvoie au cas de cette cette petite fille musulmane accompagnée par un jeune enseignant-stagiaire de l'école primaire qui se pose la question si elle ne se sent pas « mal à l'aise par moments d'être suivie par un jeune adulte homme alors que ses parents lui ont dit de ne pas s'entretenir avec un homme étranger ». On peut supposer les fantasmes qui jouent, les résistances et les interpellations qui fonctionnent. À d'autres moments cette jeune fille se sent débordée par une matière et elle décroche : « elle met ses affaires dans son cartable et ne fait plus rien... pas moyen de la motiver à travailler avec moi ", raconte l'enseignant qui l'accompagne dans son processus d'inclusion scolaire. La petite change seulement d'avis si une enseignante l'invite à travailler. Frustration énorme pour ce stagiaire en enseignement spécialisé ! C'est l'imaginaire qui touche au réel. Son manque l'incite à travailler au niveau symbolique.

\section{Le réel du corps comme défi}

Difficile dans le travail inclusif est donc le cas des enfants qui sont débordés par la jouissance. C'est le non-savoir, le réel qui peut mettre les jeunes enseignants mal à l'aise. C'est par exemple le cas pour les enfants qui sont dits " être en retard "et qui ne maitrisent pas leur corps, qui envahis par la jouissance souffrent par exemple d'énurésie. Les causes d'un tel symptôme peuvent être multiples. L'enseignant ou tout autre intervenant peut s'éprouver touché par des affects dont lui aussi jouit alors à sa manière. Marqué par le dégout, une future enseignante spécialisée refuse de laver un enfant qui n'est pas propre à l'âge de 5-6 ans. Sans pouvoir aller dans le détail, nous pouvons dire que c'est son rapport au corps et à l'autre qui est en question ici.

Beaucoup sont confrontés à des enfants en agitation " continuellement en mouvement dans une classe tout en risquant de se faire mal, qui dérangent les autres enfants dans leur travail " et qui “entrent très vite dans une spirale dangereuse pour chacun dans la classe». Difficile de ne plus considérer un tel enfant comme sujet mais comme objet réel, " happé par le vide de l'Autre », à la recherche d'une place symbolique (Forget, 2017, p. 84-85). Dans ce cas, c'était l'enseignante elle-même qui finissait par se sentir sans repères symboliques pour faire avec la situation de son élève en souffrance.

Une autre enseignante se voit défiée par un jeune pour qui la jouissance semble être au poste de commande. C'est l'objet toxicomane réel qui semble pallier l'absence de lien à l'Autre. Mais c'est aussi dans “ son corps négligé, nauséabond qu'il approche de près les autres et les angoisse».

\section{* Altération de l'image, déchirure et altérité}

De telles expériences affectent les enseignants. Ils se demandent à quelle place ils sont mis et s'ils peuvent assumer ce métier qu'ils désiraient ou voulaient faire. 
Certains évitent à ce qu'ils soient touchés dans leur chair par la parole de l'autre. C'est ainsi qu'un enseignant dit à un autre : "Tu n'as qu'à être très sec avec celui-là ! ” Ils entrent dans la dénégation, ce vouloir ne rien vouloir savoir (Vasse, 2004, p. 317) : "Je ne suis quand-même pas ami avec elle, je ne vais quand-même pas l'épouser! ". C'est la méfiance, le mépris, le regard accusateur de l'Autre qu'on projette éventuellement dans une classe où " une fille qui par sa biographie ne fait que prendre des distances" par rapport au stagiaire et lui flanque au visage : "qu'elle ne s'intéresse pas à son désir de la faire avancer ». De telles expériences délogent le jeune stagiaire de l’image qu'il s'est faite de lui-même grâce aussi à ses refoulements.

Confronté à la fragmentation de l'image que nous avons du corps de l'autre, l'image du corps propre peut se morceler en miroir. Nous sommes interpelés et c'est l'angoisse qui peut en jaillir. Aussi le fait qu'un autre « se colle à notre corps, peut induire des angoisses qui viennent d'expériences de notre passé „ comme l'affirme un enseignant. C'est le rapport au corps propre que le jeune enseignant-stagiaire en classe d'inclusion est interpellé à travailler. En effet le corps, c'est ce que nous avons de plus intime, mais aussi de plus étranger, c'est ce dont nous jouissons, avons peur, honte ou fierté. Il est parlant et a besoin de la parole de l'Autre.

La confrontation à un discours normé, scientifique peut lui aussi bloquer le stagiaire en l'invitant à objectiver le sujet élève, donc à se construire une image de l'élève en question et à s'y enfermer. C'est le cas si quelqu'un lui dit: "cet enfant a de toute façon un retard de 32 mois " ou "si on savait au moins qu'il est autiste " Se pose la question quel motif et quelle violence peuvent se cacher derrière une telle affirmation. Qu'en est-il de l'enfant, sujet de désir? Est-ce que cette représentation n’encombre pas la Vie, le réel comme impossible à représenter? Quel rapport au savoir est en jeu ici? Comment s'en rendre compte et comprendre l'effet sur l'enfant en question. C'est surtout problématique pour un jeune enseignant d'être confronté à de tels propos. Comment lui permettre de ne pas s'enfermer dans une image " scientifique " de l'autre, de ne pas se laisser enfermer dans une passion de la causalité (Gori, 2002) pour passer à une passion de l'ignorance empêchant ainsi son processus de formation dans ce métier de l'humain?

Et finalement comment rencontrer les parents d'enfants en difficultés? Comment se confronter en tant qu'enseignant stagiaire par exemple avec un discours le culpabilisant? Comment faire avec des discours ambiguës de parents tiraillés parfois entre la volonté de faire le bien pour leur enfant, à l'appui du discours de l'inclusion et le protéger trop, d'autre part. Évoquons à ce sujet une situation où l'enseignant partage avec les élèves sur la façon dont ils peuvent inclure et soutenir un enfant en difficulté. Or les parents ne supposent pas leur enfant suffisamment sujet pour être présent en classe lors de cet échange sur lui.

Toutes ces expériences touchent les stagiaires au corps. Ils sont délogés dans leur image et dans les discours qu'ils tiennent. Parfois ils ont peur de parler, de dire leur ressenti. Ils ne veulent pas entrer en conflit avec les élèves, les parents, les formateurs 
qui les accompagnent sur le terrain, parce que cela les emmènerait à avouer leur “propre conflit intérieur " Vasse, 1981, p. 156). L'un ou l'autre préfère ainsi " faire le mort ", afin de ne pas être blessé narcissiquement. Et en effet certains enseignants se sentent débordés, se découvrent dans la haine, le désir de mort liés à des sentiments de culpabilités et à des passages à l'acte. La découverte de l'altérité extérieure et intérieure constitue aussi une chance de progresser.

\section{* L'altérité et « l'inquiétante étrangeté » comme défis pour la formation des enseignants}

Les jeunes stagiaires viennent avec leurs fantasmes, identifications, leurs idéaux et objets internes à partir desquels ils se sont construits. Carlo, enseignant-stagiaire, “ commence à trembler, à transpirer fortement s'il se trouve devant une classe ». C'est la frayeur! On peut même parler d'inquiétante étrangeté. En effet dans la confrontation avec les élèves des signifiants nécessaires manquent ou s'échappent (Freymann, 1999, p. 2015). Le monde qu'il a connu jusqu'alors n'est plus le même. Nous avons rencontré de jeunes enseignants, qui en tant qu'adolescents vivaient dans leur monde restreint, dans une certaine protection, voire solitude, et que là, en fonction, tout d'un coup se retrouvent confrontés à d'autres mondes.

Pour Freud “ l’inquiétante étrangeté prend naissance dans la vie réelle lorsque des complexes infantiles refoulés sont ranimés par quelque impression extérieure ou bien lorsque de primitives convictions surmontées semblent de nouveau être confirmées » (Freud, 1933). Sans réduire “ l'Unheimlich » à cet aspect, c'est la violence, tel ou tel trait physique, l'être de l'autre avec qui il faut faire avec, sans nécessairement le comprendre. Il importe donc au formateur de terrain de faire en sorte que le jeune stagiaire ne " s'engouffre pas dans un trou d'abîme où la souffrance côtoie la jouissance " (Cabassut, 2005, p. 11). Évoquons encore une situation, où les élèves d'une classe inclusive sont très incommodés par le comportement d'un condisciple. Ils vivent là cette relation comme " unheimlich ». Ils préféreraient qu'il soit exclu. Les enseignants le soutiennent à s'inclure et à faire avec son mal. Ces efforts sont jugés par les autres élèves comme une manque d'attention par rapport à leurs propres besoins de reconnaissance et leurs malaises. Comment répondre à ces demandes? Et comment aider cet élève à avancer?

Les enseignants se demandent comment faire aussi bien avec la haine des élèves comme avec la leur. C'est l'angoisse, la “ Hilflosigkeit » qui s’installe. Comment parler en classe de cette “ inquiétante étrangeté » où les jeunes rencontrent dans le contact réel quelque chose qui leur est intime?

\section{䊅 L'usure}

Dans de telles situations l'enseignant bien intentionné se voit souvent tenté de vouloir contenir la situation à travers une panoplie de mesures, de règles et de sanctions. C'est un fantasme inconscient de maîtrise qui peut le guider éventuellement. Entrer dans un discours du maître et de la fabrication de bons élèves risque de tomber dans 
la plainte de l'impuissance et d'une certaine « usure »(Allione, 2010, pp. 67-94). Nous avons pu voir aussi avec quel engagement les enseignants désiraient que les jeunes puissent travailler leur angoisse et leur rapport à l'Autre. Par ailleurs, remarquable était d'autre part chez certains de ces jeunes enseignants le désir de se former à travers cette situation complexe et de bricoler des stratégies afin de nouer le symbolique, l'imaginaire et le réel dans le domaine professionnel.

Comment se former à faire avec l'altération qui s'installe en contexte inclusif ? C'est encore à partir de quelques expériences et interviews avec des jeunes enseignants que nous voulons proposer quelques idées.

\section{$\checkmark$ Des attitudes et compétences à développer pour l'enseignant}

Compte tenu de ces défis, le jeune enseignant est invité à développer une attitude, un style et certaines compétences qui lui permettent de travailler avec les jeunes. C'est un processus de transformation qui doit se faire souvent. Ils découvrent qu'ils se trouvent en impasse s'ils rencontrent le jeune handicapé uniquement comme quelqu'un qu'il faut " réparer » et non comme un sujet souffrant et jouissant (Russ \& Leguil, 2015, p. 120).

Le formateur de terrain a donc comme tâche d'aider le jeune enseignant à travailler la question de la jouissance, du pulsionnel, du rapport au corps et aux discours enfermant. Même si ce travail ne vise pas à “traverser le fantasme » il touche à la question du fantasme, donc la façon du jeune enseignant de traiter le réel à partir du symbolique et de l'imaginaire.

\section{Un travail sous transfert}

Travailler le rapport à l'altérité, peut débuter à partir des affects et symptômes, de la déchirure de l'image de soi qui peut parfois se produire. Cela touche à la question du transfert, parce que les enseignants comme les élèves en souffrance déplacent sur le lieu de l'école leurs désirs inconscients et touchent à leur insu l'inconscient de l'autre (Paturet, 2012, p. 43).

Ce qui importe done c'est de soutenir le stagiaire dans sa réflexion et la mise en parole, de le “ pousser » à mettre au travail la place à laquelle il se met et a mis le jeune en difficulté. Il s'agit de l'aider à interpréter certaines incidents, manifestations symptomatiques, mais de telle façon à laisser ouvert le sens. Évoquons à ce sujet un enseignant qui pensait “ que cela irait sans s'y mouiller trop, qu'il n'avait pas besoin de s'engager par rapport à un groupe ». Ce n'est qu'après que les élèves le provoquaient par leur indiscipline, qu'il se rendait “ compte de l'importance à leur donner une place de sujet » à travers sa façon de transmettre du savoir.

\section{档 «Bien-dire » versus dire le bien}

Plutôt que de dire le Bien, qui serait la position du discours du maître, le formateur favorise le “Bien-dire " (Lacan, 1974, p. 65), c'est-à-dire l'énonciation du jeune 
enseignant dans sa démarche d'être de désir et de sujet divisé dans le contexte en question. Lors des échanges, ce dire est justement attendu au lieu d'achoppement du discours du jeune stagiaire : là où ça m'échappe, là dois-je advenir comme sujet. La tâche de l'accompagnateur du jeune stagiaire est alors de soutenir et relancer ce " Biendire " pour qu'il puisse lâcher le plus possible sur ses résistances et sur sa position de maîtrise, de savoir avec certitude comment faire (Pigeon, 2019). Il s'agit d'aider l'enseignant à passer d'une position d'impuissance à celle du métier d'impossible.

Lors d'une supervision, une formatrice de terrain parle d'un élève qui durant tout un temps n'arrêtait pas de faire des crises : “jette des objets sur les autres enfants, parfois des chaises et attaque l'enseignante ». Il s'agit d'un enfant qui à la suite du décès d'un proche remplace la parole par l'acte, il n'obéit plus à l'enseignante stagiaire, ne s'intègre plus dans ce collectif et sème plutôt le désordre en classe. Cette situation demandait à la formatrice un très long travail de mise en parole et d'écoute pour autoriser la stagiaire à se dégager des pièges “ de la représentation et de l'exaspération de l'imaginaire » (Vasse, 1978, p. 217) Il s'agissait de la soutenir à ce qu'elle vise avec son désir le réel, au-delà de l'imaginaire destructeur.

\section{Travailler le cadre}

Nous savons comment l'enfant dit hyperactif peut vite déranger un cours. C'est tout à fait compréhensible que vivre dans un collectif a un important côté pulsionnel, jouissif, la situation peut vite tourner au chaos. Le jeune perturbateur peut être considéré ainsi comme intrus et risque être rejeté par ses copains.

Approcher les jeunes, hyperactifs ou psychotiques dans leurs angoisses, troubles et agirs ne peut pas se réduire à un comportement qu'il faut cibler, circonscrire et essayer d'éradiquer d'un coup de baguette magique. Il s'agit plutôt d'élaborer des repères symboliques et imaginaires qui permettent aux enfants ou aux adolescents de se retrouver dans le social, de se calmer et de se construire comme sujet. C'est aux enseignants et éducateurs de faire cadre, de les aider à assumer une place symbolique si c'est possible, de bricoler éventuellement avec des rituels, des bouts de parole ou des jeux pour accueillir une jouissance débordante.

Comment constituer un bord? Le bord à constituer est entre réel et savoir, entre une jouissance qui déborde et le champ signifiant où il s'agit d'en dire une part. Ce bord, Lacan l'a nommé : fonction de la lettre. Ainsi peut se réduire pour le sujet le poids du sens, ce qui peut permettre à la violence de céder la place à la parole. Faire bord c'est introduire le sujet à la dimension du semblant, comme dans un cas où un élève qui pouvait être très violent a appris à se tranquilliser grâce au " calme », c'està-dire un rituel qui lui permet de se retirer du groupe, dans un lieu à lui.

\section{Des enseignants et des élèves dans des institutions « pas toutes »}

Les enseignants nous disent qu'il importe aussi, qu'eux-mêmes «puissent faire avec des situations chaotiques ». Rien de plus nécessaire que d'être ouvert à se laisser 
conduire par la Vie, la parole et l'écoute de l'autre. "Il faut parfois savoir ne pas insister, pour qu'il retrouve son calme... il faut lui dire aussi ce qu'on ressent s'il est tellement violent ou lui demander d'exprimer ce qu'il ressent. "Il importe donc de créer une atmosphère, qui ne se construise pas sur un modèle institutionnel plutôt paranoïaque, et par conséquent trop perturbant pour des enfants à traits psychotiques ou violents. Le savoir ne peut pas être le monopole des enseignants.

\section{档 La formation : advenir comme sujet professionnel}

La formation d'un enseignant de l'école inclusive d'aujourd'hui ne concerne pas uniquement l'apprentissage de gestes professionnels, comme on dit dans le langage pédagogique actuel, mais de subjectiver ce métier, c'est-à-dire de travailler sur les discours, représentations et les scénario inconscient qui nous guident inconsciemment dans ce métier. Prenons l'enseignante qui devient agressive et met un enfant après l'autre devant la porte, parce que ses élèves ce jour-là ne font pas comme elle le souhaite. Étonnée, car elle affirme que « Nomalerweise hängen die Kinder an meinen Lippen ", traduit “normalement les enfants sont suspendus à mes lèvres », elle vit leur comportement comme une attaque. Elle se sent blessée. Même s'il ne s'agit pas d'une situation catastrophique, on peut se poser la question de quel fantasme " se nourrit » sa façon d'être enseignante. Quel objet petit a, est cause du désir d'enseigner? Est-ce qu'elle se comprend inconsciemment comme sein?

Nous avons interviewé d'autres enseignants qui se voyaient au bord “du burnout» parce qu'ils " ne supportent les devoirs criblés de fautes». Eux aussi vivaient ces fautes comme une sorte de projectile qui les atteignent dans l'image qu'ils s'étaient construits de leur profession et d'eux-mêmes comme enseignant.

Râler, engueuler les élèves et installer des mesures draconiennes dans une classe est une option qui tournera normalement au pire. Elle ne fait qu'enfermer l'enseignant dans son fantasme. Être agacé, exaspéré par l'inattention, les fautes grammaticales, le refus de travailler, constitue une occasion de questionner son propre rapport à la loi de la parole, à son désir d'enseigner, peut-être aussi au mépris et à la méprise vis-à-vis de soi-même, des élèves ou du métier. Tout cela nécessite des espaces - temps de médiation, où les enseignants en formation et autres peuvent se parler, se risquer pas seulement pour survivre dans leur métier, mais y vivre vraiment. C'est un travail sur la sa propre jouissance propre à travers l'ouverture à la parole, donc à l'Autre.

Or nous risquons aujourd'hui de nous assujettir à des techniques qui anéantissent tout notre pouvoir créateur. Dans ce sens le risque « d'oublier toute éthique est peut-être le seul risque qu'il faut vraiment chercher à prévenir »(Russ \& Clotilde, 2015, p. 121).

\section{We Pour une éthique de la rencontre}

Dans le monde professionnel on s'est habitué à parler de déontologie. La vie collective à l'école a besoin de normes auxquelles le professionnel et les élèves se 
soumettent. Mais nous savons aussi que ce qui est bien pour l'un peut être mal pour l'autre. Et les normes que nous émettons se basent souvent sur ce que nous nous imaginons être le bien pour l'autre, la communauté et nous-mêmes. Une approche éthique par contre est animée par notre désir qui vise le réel, l’irreprésentable, l'impossible comme Kant l'avait déjà théorisé et comme Lacan, Zizek et Zupancic (2011) l'ont interprété et développé. Finalement ce rapport au réel se traduit par notre foi ou notre espérance en la Vie. Cette foi se constitue par un acte de liberté, mais fonde finalement aussi notre liberté et l'ouverture à l'Autre et à notre propre altérité. Une telle approche d'éthique permet une rencontre vraie entre êtres humains : “ La vie n'est pas donnée comme un objet. Elle n’est que donnée dans la rencontre. »(Vasse, 2013, p. 512).

L'élève que le jeune enseignant rencontre n'est pas identique à sa figure, à la souffrance ou les émotions que nous imaginons. C'est un Autre, un " parlêtre ». Le rencontrer ce n'est pas une affaire d'apprentissage, mais de positionnement, donc de rapport à la jouissance, à la vérité singulière, c'est une question de choix éthique. Certains élèves à besoins spécifiques n'ont pas toujours les mots pour dire, se dire, s'exprimer sur ce qui les occupe. C'est « la confiance des accueillants dans ce que parler implique »(Lebrun, 2017, p. 304) qui les aide à se dire.

Ce n'est qu'au travers de la rencontre avec un autre réel, qui sait endosser les déceptions de la vie, la perte de jouissance, et qui sait aussi « donner chair vivante à la possibilisation de l'impossible ", que le jeune peut progressivement assumer à supporter la négativité qui fait partie de la condition humaine (Lebrun J.-P., 2004, p. 78). C'est grâce à la découverte que l'existence est viable, malgré les manques et les abîmes que le "parlêtre » rencontre, que les jeunes peuvent espérer de développer leurs capacités, opportunités et advenir en tant que sujet.

L'enseignant c'est quelqu'un qui aide les enfants à découvrir la voie du désir. Dans ce sens, une étudiante en stage parle d'un enseignant qui lui a transmis qu'un élève qui a raté par exemple un devoir en classe ne doit être pas identifié à l'échec : «il y a toujours un arrière-fond ", done du réel qui fonde un espace de liberté. Or la liberté fondamentale constitue un consentement à la Vie, à “l'ex-sistence ». Elle se fait à travers la rencontre avec quelqu'un d'autre, comme le déni de la vie va être aussi une manière de ne rencontrer personne (Vasse, 2005). "Pour marcher comme pour parler, l'homme doit franchir un seuil et il ne peut le faire que relativement à un appel, qui est promesse de rencontre. Du désir de la rencontre, étayé sur la promesse qu'elle aura lieu, naît la possibilité de prendre le risque du vide, du saut, de la séparation d'avec l'image de soi. Il n'y a de rencontre véritable qu'à ce prix. " (Vasse, 2007). Mais cela présuppose que l'accompagnateur de jeunes en difficultés ait travaillé et accepté sa propre altérité et l'aime suffisamment. À cet effet il a besoin de l'écoute, de l'écho de collègues, de dispositifs d'analyse de pratiques voire de supervision. 


\section{We Conclusion}

Pour les jeunes enseignants ou stagiaires, travailler avec des enfants ou des adolescents en souffrance n’est pas évident. S’il ne faut pas nécessairement avoir une approche psychanalytique pour rencontrer l'élève, des dispositifs d'analyse des pratiques inspirés par la psychanalyse peuvent aider les jeunes enseignants en stage à reconnaître dans le jeune en face un “ je à advenir » et à travailler à ce propos leurs transferts, résistances et fantasmes.

\section{Références}

Allione, C. (2010), La part du rêve dans les institutions, régulations, supervision, nalyse des pratiques. Paris : Les Belles Lettres.

Assante, V. (2000). Situations de handicap et cadre de vie, Paris, éditions des Journaux officiels (Avis et rapports du conseil économique et social).

Cabassut, J. (2005), Le déficient mental et la psychanalyse. Nîmes : Champ social Éditions.

Dufour, D.-R. (2013). L'art de réduire les têtes, Sur la nouvelle servitude de l'homme libéré l'ère du capitalisme total. Paris : Denoël.

Ebersold, S. (2009, Nr. 61), Autour du mot: Inclusion. Recherche et formation, pp. 71-83.

Freud, S. (1933), L’inquitante étrangeté in Essais de psychanalyse appliquée. Paris : Gallimard.

Freymann, J.-R. (1999),Introduction à l'écoute. Strasbourg: Arcanes.

Forget, J.-M. (2017), Les enjeux des pulsions. La clinique des pulsions, une clinique actuelle. Toulouse : Érès.

Garel, J.-P. (2010), De l'intégration scolaire à l'éducation inclusive : d'une normalisation à l'autre. Journal des anthropologues 122-123, 143-165.

Gori, Roland (2002). Logique des passions, Paris : Denoël

Han, B.-C. (2016), Die Austreibung des Anderen. Gesellschaft, Wahrnehmung und Kommunikation heute. Frankfurt am Main : S. Fischer.

Konferenz, K. (2015, März 18), https://www.kmk.org/fileadmin/Dateien/veroeffentlichungen beschluesse/2015/2015 03 12-Schule-der-Vielfalt.pdf.

Lacan, J. (1974), Télévision. Paris : Seuil.

Lebrun, L. a.-P. (2017),Une parole pour grandir. Suivi de Edipe empêché. Toulouse : Érès.

Lebrun, J.-P. (2004, no 12), Une forclusion de la rencontre. "Essaim », pp. 73-83.

Melman, C. (2002), L’homme sans gravité. Paris : Denoël.

Paturet, J.-B. (2012), Le transfert à l'usage des enseignants et des éducateurs. Approche psychanalytique. Montpellier : Sauramps Médical.

Pigeon, M. (2019, Juillet 2), L'éthique du bien dire. Récupéré sur Psychasoc: http://Www. psychasoc.com/Textes/L-ethique-du-Bien-dire

Russ, J., \& Clotilde, L. (2015), La pensée éthique contemporaine, Paris : Puf.

Vasse, D. (1978), Un parmi d'autres. Paris : Seuil.

Vasse, D. (1981), L'ortophoniste pris aux mots. Rééducation orthophonique, 153-164.

Vasse, D. (2004). La grande menace. La psychanalyse et l'enfant, Paris : Seuil. 
Vasse, D. (2007, Mai). La souffrance : altération, altérité. Christus no HS -, pp. 113-127.

Vasse, D. (2005), Naître pour la mort - Naître à la vie. Récupéré sur https://www.denis-vasse. com/naitre-pour-la-mort-naitre-a-la-vie.

Vasse, D. (2013), Agathe ou la jumelle occultée. La cure d'une petite fille autiste. Montrouge : Bayard. Zupancic, A. (2002), Ethics of the Real Kant and Lacan, London, New York: Verso. 